\title{
Evolution of deformation and breakage in sand studied using X-ray tomography
}

\section{Z. KARATZA* $*_{\ddagger}$, E. ANDÒ + , S.-A. PAPANICOLOPULOS $\dagger$, J. Y. OOI† and G. VIGGIANI}

\begin{abstract}
Particle breakage of a granular material can cause significant changes in its microstructure, which will govern its macroscopic behaviour; this explains why the mechanisms leading to particle breakage have been a common subject within several fields, including geomechanics. In this paper, X-ray computed micro-tomography is used, to obtain three-dimensional images of entire specimens of sand, during high-confinement triaxial compression tests. The acquired images are processed and measurements are made on breakage, local variations of porosity, volumetric strain, maximum shear strain and grading. The evolution and spatial distribution of quantified breakage and the resulting particle size distribution for the whole specimen and for specific areas are presented here for the first time and are further related to the localised shear and volumetric strains. Before peak stress is reached, compaction is the governing mechanism leading to breakage; neither compressive strains nor breakage are significantly localised and the total amount of breakage is rather low. Post peak, in areas where strains localise and breakage is present, a dilative volumetric behaviour is observed locally, as opposed to the overall compaction of the specimen. Some specimens exhibited a compaction around the shear band at the end of the test, but there was no additional breakage at that point. From the grading analysis, it is found that mainly the grains with diameter close to the mean diameter of the specimen are the ones that break, whereas the biggest grains that are present in the specimen remain intact.
\end{abstract}

KEYWORDS: deformation; particle crushing/crushability; sands

\section{INTRODUCTION}

There are many examples in the literature (e.g. Roscoe et al., 1958; Bolton, 1986; Colliat-Dangus et al., 1988; Nakata et al., 2001; Coop et al., 2004; Arslan et al., 2009) that present a detailed analysis of grain breakage from triaxial compression experiments performed on sand. When a loose sand is sheared it contracts and the grains start to slide and roll relative to each other; when it cannot contract any longer, it starts to dilate. This dilatancy will produce additional compaction potential to the material and, should the imposed stresses be sufficiently high, then particles start to break. Breakage during loading will cause an evolution in the grain size distribution, essentially changing the material's properties (e.g. compressibility, shear strength and fabric) and therefore the critical states will not be the same as for the intact material (e.g. McDowell et al., 1996; Tengattini et al., 2015). These studies contribute greatly to the understanding of how particle breakage affects the behaviour of granular materials at the macro scale. Making a link between the microscopic changes caused by breakage or dilatancy and the bulk response of a material is still quite challenging.

Continuum constitutive models attempt to predict the evolution of breakage and/or strain localisation, taking into account experimental data regarding the bulk response

Manuscript received 8 August 2016; revised manuscript accepted 11 April 2017. Published online ahead of print 13 July 2017.

Discussion on this paper closes on 1 July 2018, for further details see p. ii.

Published with permission by the ICE under the CC-BY 4.0 license. (http://creativecommons.org/licenses/by/4.0/)

* School of Engineering, The University of Edinburgh, Edinburgh, UK (Orcid:0000-0002-4083-6502).

$\uparrow$ School of Engineering, The University of Edinburgh, Edinburgh, UK.

† Univ. Grenoble Alpes, CNRS, Grenoble INP, Laboratoire 3SR, Grenoble, France. throughout the test or experimental observations during the unloaded and post-testing state of the specimen. However, the models can be largely empirical and might only relate to the specific cases being studied, so the need to understand the micro-mechanisms of grain breakage under various loading regimes is vast.

Kikumoto et al. (2010) explained how the broadening of the grading will shift the critical state line and compression line downwards in the compression plane, and have developed a constitutive model that incorporates the changes due to particle crushing. Tengattini et al. (2016) explained why the critical state of sand is non-unique, when expressed in terms of stress and void ratio. They used a thermodynamically consistent framework to predict the critical state and its dependence on breakage, rather than fitting experimental data, and introduced a new breakage mechanics model. These examples confirm the need to quantitatively describe breakage during a test, in order to provide quantitative information to facilitate the development of constitutive modelling and overall understanding.

X-ray computed micro-tomography (XCT) has enabled the observation and measurement in three dimensions of the deformation and kinematics of materials during loading. Even though XCT has been used extensively as a medical diagnostic tool since the 1970s, it has only recently been used as a research tool in geomechanics (e.g. Desrues et al., 1996). In recent studies about grain breakage, there is an interplay of image resolution, number of particles and specimen size.

Zhao et al. (2015) investigated the single particle fracture behaviour (shape evolution and breakage energy) of two different types of natural sand, and acquired images with a resolution of a few micrometres (nanoXCT). They showed a connection of the initial heterogeneity of the microstructure of the particles (e.g. internal voids) with the type of fracture and the shape and size of fragments. Such detailed study is nearly impossible to perform on a full specimen, because 
there are significant limitations in image resolution as well as the computational time needed to perform measurements on each particle. Cil \& Alshibli (2014) studied the evolution of sand fracture under one-dimensional (oedometric) compression. They studied the effect of the slenderness ratio of a loose sand assembly with uniform grading, used images from synchrotron to visualise the grains (number, position and deformation), and then made a qualitative comparison of the deformation process with a discrete-element method (DEM) model. Their observations from the images are very useful and qualitatively validate the DEM; however, no quantitative measurements have been made from image processing. Furthermore, the relatively small number of particles in the oedometer is such that it posed a serious question regarding whether this is a representative specimen or just an assembly of grains. Oda et al. (2004) observed changes in the microstructure of a shear band from thin sections post-mortem and supplemented the experimental observations by using XCT. Plane strain tests, with constant minor principal stress, were performed on a dense, wellgraded sand and they observed a dilatant shear band, but could not make any observations on particle orientations or produce any quantitative data. Fonseca et al. (2013a, 2013b) published a study regarding the quantification of soil fabric using both directional and scalar parameters, coming from high-resolution XCT and scanning electron microscopy (SEM) images of reconstituted sub-samples, taken from a specimen under conventional triaxial testing. They showed the importance and relevance of soil fabric to the mechanical response of a sand under triaxial loading. To achieve the high resolution required, different specimens and sub-samples had to be used for each loading stage, so some kind of reconciliation of the differences between different samples had to be performed to interpret the results. It is naturally advantageous to be able to study the evolution of fabric in one specimen. Gkiousas-Kapnisis et al. (2015) used XCT images of well-graded Hostun sand under triaxial loading (described in Alikarami et al. (2015)), to study the evolution of grading. The angularity of the grains and the grading of the sand created important challenges during image processing, especially regarding the segmentation of the particles and the definition of a size descriptor to obtain the mean diameter of the particles. Andò et al. (2013) describe the challenges to overcome, regarding granular mechanics and image processing.
They attempted to improve on previous work with image correlation and tracking of individual intact grains and an approach to identify and characterise contacts. Regarding the identification of contacts, improvements can be made, due to possible inaccuracies resulting from the resolution of the images and the segmentation algorithm.

The main focus of this work is to study the grain-scale changes that an assembly of grains undergoes during loading, and therefore XCT is used, to obtain three-dimensional (3D) images of entire specimens of sand, during loading, under relatively high-confinement $(7 \mathrm{MPa})$ triaxial compression tests. The acquired images are processed and measurements are made on breakage, local variations of porosity, volumetric strain, maximum shear strain and grading. The evolution of breakage and the resulting particle size distribution, for the whole specimen and for specific areas, are presented for the first time and are later related to the localised maximum shear and volumetric strains. To avoid some of the problems mentioned previously, uniform specimens of sand with very rounded particles are used to facilitate a clear distinction between intact and broken particles.

\section{TESTED MATERIAL}

The material used for this study is Caicos ooids, provided by ExxonMobil; this is a sand with very rounded particles (see Fig. 1) that grow from fragments of corals, coming from Ambergris Shoal in the Caicos platform, in the British West Indies. Ooids are ellipsoid concretions of calcium carbonate and aragonite crystals, which form in marine environments (rich in carbonate calcium) by gaining successive layers (smooth, uniform carbonate coatings) around a nucleus; during this process there is a resting period before the addition of each new layer (Lloyd et al., 1987). Depending on the successive growth and resting periods, variations in the thickness of the coatings and the appearance of the nuclei can be expected, altering the internal porosity of each grain and potentially its strength and stiffness.

The ooids have a mean diameter $\left(D_{50}\right)$ of $320 \mu \mathrm{m}$. To ensure that breakage and the associated plastic deformation can be discerned with greater clarity, the sand was sieved (opening of $315 \mu \mathrm{m}$ ). This resulted in the test material having a $D_{50}$ of $380 \mu \mathrm{m}$ and a coefficient of uniformity $\left(C_{\mathrm{U}}=\right.$ $\left.D_{60} / D_{10}\right)$ equal to $1 \cdot 19$.

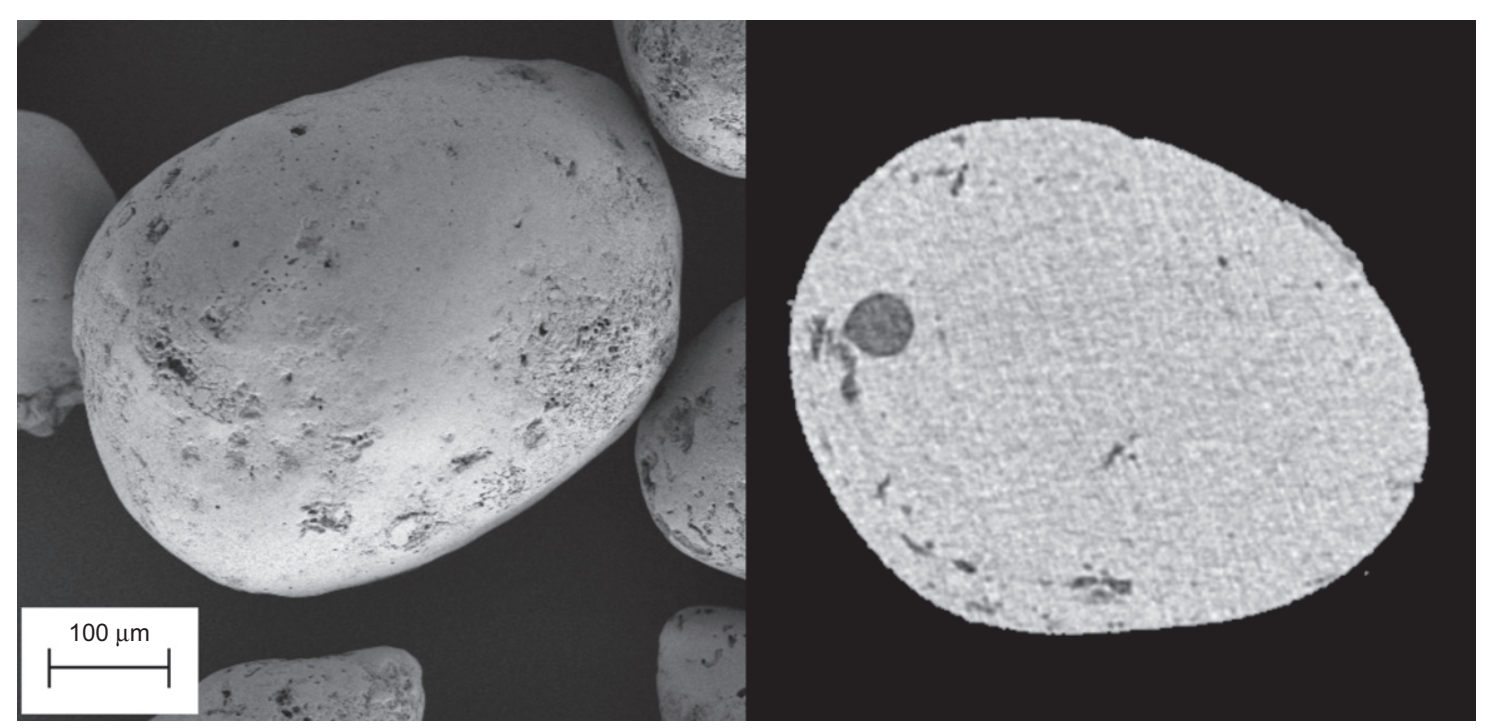

Fig. 1. SEM of the surface of a grain (courtesy of Anita Torabi, University of Bergen - left) and a vertical slice of the volume of a grain from nanoXCT (courtesy of Budi Zhao, City University of Hong Kong - right) 
Table 1. Experimental programme

\begin{tabular}{l|c|c|c|c|c|c|c}
\hline Test* & $\begin{array}{c}\text { Height: } \\
\mathrm{mm}\end{array}$ & $\begin{array}{c}\text { Diameter: } \\
\mathrm{mm}\end{array}$ & $\begin{array}{c}\text { Confinement: } \\
\text { MPa }\end{array}$ & $\begin{array}{c}\text { Initial relative } \\
\text { density: } \%\end{array}$ & $\begin{array}{c}\text { Initial } \\
\text { porosity: } \%\end{array}$ & $\begin{array}{c}\text { Voxel size: } \\
\mu \mathrm{m}\end{array}$ & $\begin{array}{c}\text { Number of } \\
\text { scans }\end{array}$ \\
\hline TCXL $\dagger$ & $24 \cdot 2$ & $10 \cdot 7$ & $0 \cdot 1$ & 46 & $35 \cdot 2$ & $15 \cdot 57$ & 18 \\
TCR & $22 \cdot 3$ & $10 \cdot 1$ & 7 & 47 & $35 \cdot 0$ & N/A & N/A \\
TCX1 & $23 \cdot 5$ & $9 \cdot 4$ & 7 & 58 & $38 \cdot 8$ & $15 \cdot 57$ & 7 \\
TCX2 & $23 \cdot 3$ & $9 \cdot 9$ & 7 & 54 & $38 \cdot 4$ & $15 \cdot 50$ & 6 \\
TCX3 & $23 \cdot 4$ & $9 \cdot 3$ & 7 & 57 & $8 \cdot 82$ & 2 \\
\hline
\end{tabular}

*TC, triaxial compression; X, with XCT; L, low confinement; R, reference test.

$\dagger$ The results of test TCXL have already been presented in Andò et al. (2012), where the test was named COEA01.

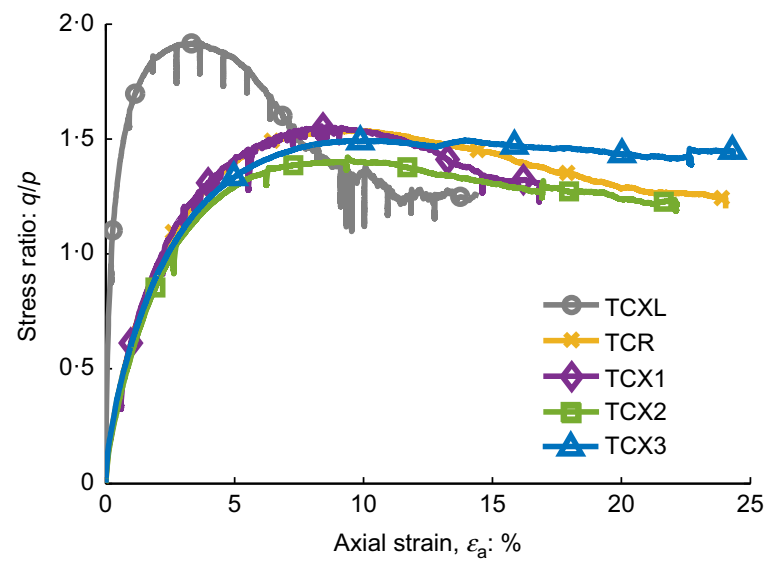

(a)

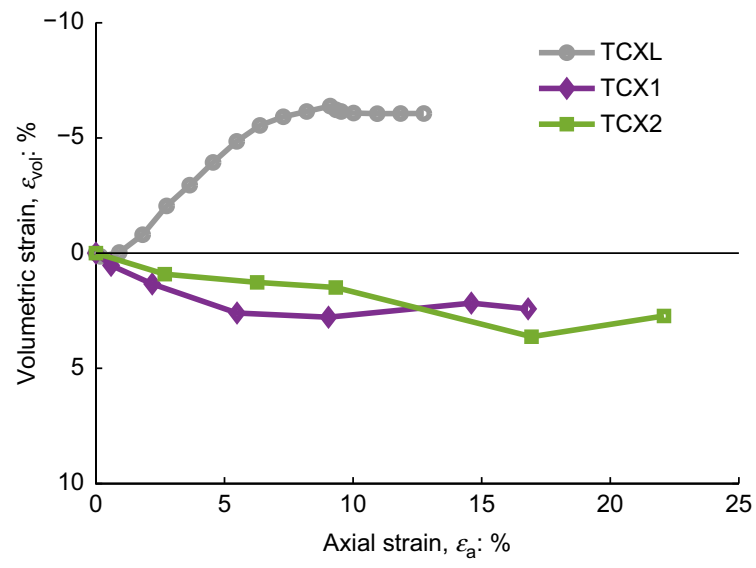

(b)

Fig. 2. Macroscopic response of Caicos ooids: (a) stress ratio plotted against axial strain (triaxial response); (b) volumetric strain plotted against axial strain (image-based strain measurements)

\section{EXPERIMENTAL CAMPAIGN \\ Apparatus and testing programme}

An experimental campaign of several relatively highconfinement triaxial compression tests was performed, a selection of which is presented here (details provided in Table 1). To allow the acquisition of images with XCT, the loading apparatus has to be transparent to X-rays and both the apparatus and the specimen must have small dimensions to allow the X-ray's cone beam to obtain a sufficient resolution. The triaxial cell and the specimen preparation method used here are described in Alikarami et al. (2015). Specimens are pluviated into a neoprene membrane, stretched in a mould; the membrane's contribution to the lateral stress has been accounted for, as described in Bishop \& Henkel (1962). During XCT (detailed in the following section), the specimen must not be straining or changing in any way, and therefore the loading is paused for the duration of the image acquisition (indicated by the axial stress relaxations that can be observed in Fig. 2(a)). Each pause is labelled by a number in order to mark different loading stages (LS\#). The loading was achieved by moving the bottom platen upward, with a displacement rate of $21 \mu \mathrm{m} / \mathrm{min}$ (axial strain rate of $0 \cdot 1 \% / \mathrm{min}$ ). It should be mentioned that the specimens of tests TCXL and TCR have a less uniform grading in comparison to the rest and were created from the original Caicos sample with a $D_{50}$ of $320 \mu \mathrm{m}$. The minimum and maximum void ratios have been experimentally determined (using a vibratory table (ASTM D4253-14 (ASTM, 2014))) and are $e_{\min }=0.63$ and $e_{\max }=0.92$ respectively, from which the initial porosity and relative density (Table 1) were calculated (using the bulk density and the grain density) and used to validate the measurements deriving from the $\mathrm{XCT}$ images.

\section{Macroscopic loading response}

The macroscopic responses are presented in Fig. 2. The stress ratio (stress deviator $q=\sigma_{1}-\sigma_{3}$ over mean stress $\left.p=\left(\sigma_{1}+2 \sigma_{3}\right) / 3\right)$ is plotted against the axial strain (shortening); both these measurements came from direct recordings from the load cell and a displacement gauge. The total volumetric strain (calculated from the end of the isotropic compression, where $q=0 \mathrm{MPa}$ ) is plotted against the axial strain; the former was calculated from binary images and therefore there is no curve for the test where no XCT was performed throughout the test.

The experimental procedure is fairly repeatable, both in initial relative densities and in macroscopic stress-strain responses. It is also clear that TCXL, which was tested at $100 \mathrm{kPa}$ confinement, shows a higher peak of the stress ratio, and post peak all specimens reach a similar stress value (plateau) at the end of the test. As expected, with a higher confinement and additionally a looser initial state of the specimen, an overall more ductile response of the material was observed. The immediate effect of the initial relative densities and the applied confinement can be seen at the volumetric against axial strain plot, where TCXL was the only highly dilatant specimen with an expected slight initial contraction. Even though specimens TCXL and TCR have similar initial grading and relative density, the much higher confinement applied on TCR caused the grains to break (as seen from a sieving analysis at the end of the test).

\section{$X$-ray imaging}

The X-ray scanner in Laboratoire 3SR, Grenoble, France (described in Andò (2013)) that was used has an X-ray source which emits a polychromatic (i.e. photons with a broad 

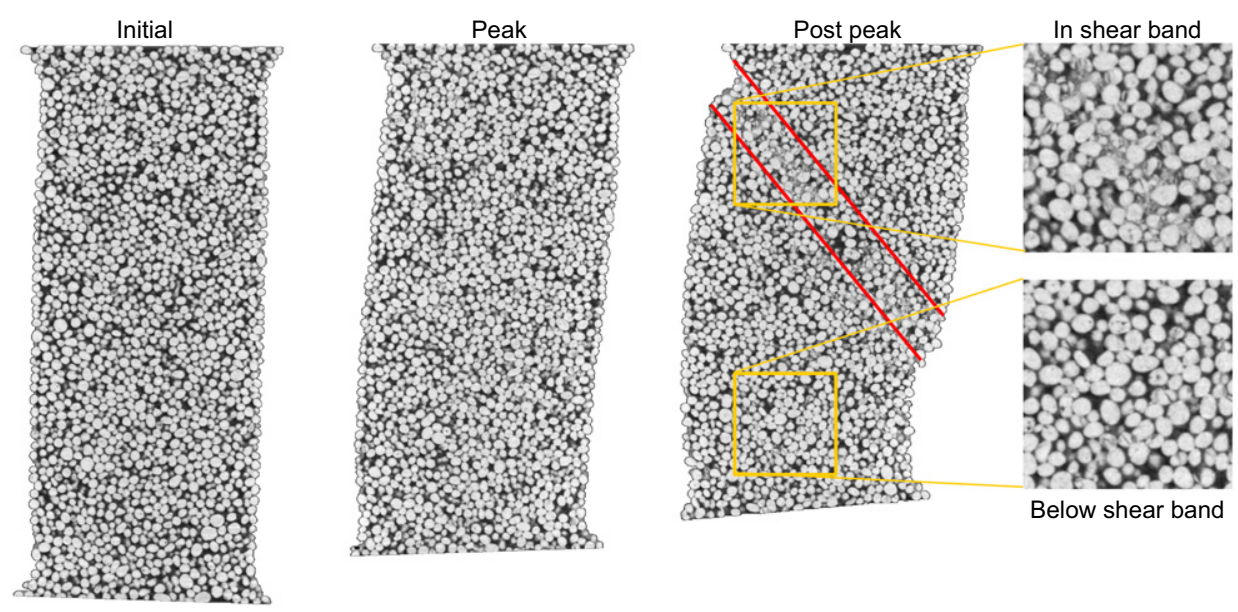

Fig. 3. Vertical sections for test TCX1 during the initial state, peak and post peak with zoomed images of regions with and without breakage

spectrum of energies) cone beam; the beam interacts with the object (here, the triaxial apparatus and specimen) that is being scanned. The photons that cross the material (i.e. are not absorbed by the material) are recorded by a detector as a two-dimensional (2D) projection of the X-ray attenuation (closely related to density) of the whole scanned object at a certain orientation. In order to obtain a 3D image of the specimen, multiple $2 \mathrm{D}$ projections at different orientations are acquired, by rotating the specimen during scanning. Wildenschild \& Sheppard (2013) described in more detail the image acquisition process and Schluter et al. (2014) presented an excellent review of the visual artefacts that can potentially limit the image processing or affect its accuracy. Fig. 3 shows vertical sections of the initial, peak and post-peak states, along with zoomed-in images relating to breakage.

\section{TEST RESULTS}

In image processing, measurements can be performed over the entirety of an imaged specimen. The measurements can be made on either a grey scale or a binary image, where the image has been separated into two distinct phases. The main benefit of image processing of a grey scale image is that there is no need to distinguish between phases, eliminating thresholding errors.

An image can be used as a whole field, where there is one result per image (bulk measurement, see $\varepsilon_{\mathrm{vol}}$ in Fig. 2(b)), but also measurements can be performed on elementary volumes within the image, to investigate local variations of a property. The bulk measurements help to validate the image processing, because they can be compared with experimental data that have not been obtained from the images. In all the aforementioned cases, if the measured property is a result of an algorithm performed directly on an image (whole or elementary volume), this will be referred to as a continuum measurement.

Usually, in granular geomechanics, the image is binarised into the solid phase and the voids. If, later on, the particles are segmented (i.e. separated from each other) and the calculations are performed on each individual grain instead of an elementary volume, then this will be termed a discrete measurement. Discrete measurements are of interest for the measurement of granular fabric, as well as the ability to track deformation (displacement and/or breakage) of individual grains. In this paper, both discrete and continuum methods are employed and compared.

\section{Porosity distribution}

Three-dimensional porosity maps were created based on the density distribution of the grey scale images. The images did not undergo a binary classification between the solid and pore phase at this stage, to avoid misinterpreting thresholding errors into the porosity calculation.

Initially, a cubic representative elementary volume (REV) was defined, then nodes were positioned throughout the image and the REVs were centred around each node. The average of the grey scale of each REV was measured, from which the porosity of each REV was calculated. The algorithm and the choice of the REV are based on previous work of Andò (2013). With this algorithm a threshold needs to be imposed to distinguish between phases; to avoid thresholding errors, the algorithm was modified to use a normalised function to calibrate the average grey value of each REV into a porosity equivalent, using the bulk porosity of the specimen that is presented in Table 1. The REV chosen in this work has a size of $2 \cdot 9 D_{50}$, which is consistent with the literature for spherical/sub-spherical uniform assemblies (e.g. Razavi et al., 2006). It should be mentioned that the limited amount of breakage did not cause the $D_{50}$ to change (as can be seen in the following sections), and so in this paper $D_{50}$ will refer to both initial and final mean particle size. In addition, the selection of the inspection cube was based on measurements performed on both the initial and final loading stages. The same size was determined for both loading stages, suggesting that the inspection cube is an REV.

In Fig. 4, cross-sections of the same orientation are presented for every loading stage of test TCX1. The bulk porosity is noted below each map as it has been evaluated from the porosity maps and from measurements of the bulk porosity from binary images. This comparison shows a maximum difference of $1.4 \%$ of the porosity, with a constant underestimation of the measurements coming from the binary images. This is due to a constant inclusion of some of the void surrounding the specimen at the REVs, at the boundaries of the specimen, which would result in a higher mean porosity than the actual.

The specimen during isotropic compression is rather homogeneous (initial state), showing a decreased porosity close to the boundaries, an effect that could have been caused by the ineffective removal, from the images, of the membrane at some areas. As the deviatoric loading progresses, there is an overall reduction of the porosity (from initially $42 \%$ to finally $37 \%$ ) at the bottom half of the specimen. At the final two loading stages, a band with increased porosity starts to appear (49\% porosity). This indicates that some dilative volumetric strains will appear in the band. At the lower right part of this band, a local reduction of the porosity can be noticed ( $35 \%$ porosity), indicating some compaction. Visual observations of the X-ray images show that most 


\begin{tabular}{l|ccccccc}
\hline Loading stage & 1 & 2 & 3 & 4 & Peak & & \\
\hline
\end{tabular}

Fig. 4. Porosity maps and values of bulk porosity for test TCX1

comminution takes place along the band (Fig. 3). Finally, the trend of the volumetric curve, as plotted in Fig. 2(b), is in accordance with the initial reduction and later on increase of the bulk porosity, as it has been measured from the binary images.

As seen in Fig. 5, specimen TCX1 (41\% porosity) was initially looser than TCX2 (37\% porosity) and even though they both exhibit an overall reduction of porosity, the distribution of the porosity in the final loading stages is rather different. At the final loading stages, TCX2 (LS5 $\left.\varepsilon_{\mathrm{a}}=22 \%\right)$ exhibits a band with lower porosity $(30 \%)$ compared to the bulk, indicating compaction in the band, as opposed to TCX1 (LS7 $-\varepsilon_{\mathrm{a}}=17 \%$ ) which indicates dilation in the band. At the same axial strain, however $\left(\varepsilon_{\mathrm{a}}=17 \%\right)$, which corresponds to LS7 and LS4 of TCX1 and TCX2, respectively, both specimens exhibit an overall compaction, with TXC1 showing some dilation in the shear band. Particles of test TCX1 break more than those of TCX2; the generation of fragments (smaller particles), along with the fact that the fragments are more angular in comparison to the intact particles, could justify the dilative volumetric behaviour along the band where most of the breakage concentrates. To provide further insights into the evolution of the shear band and the breakage of grains, and on how these relate to the volumetric and maximum shear

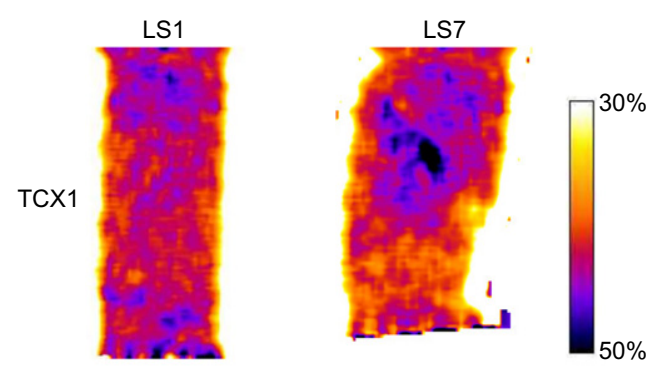

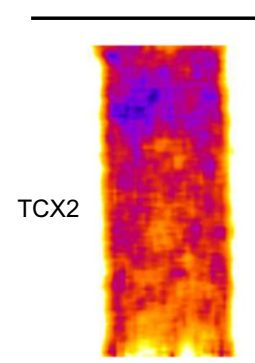

LS1

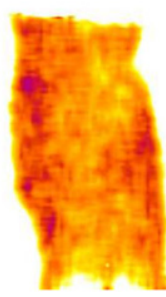

LS5

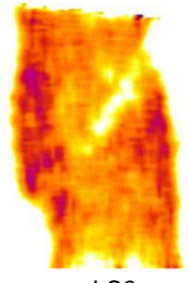

LS6
Fig. 5. Comparison of the distribution of porosity between tests TCX1 and TCX2 strains, digital image correlation (DIC) is performed, as described in the following section.

Digital image correlation

Digital image correlation is an image analysis method applied to non-contact full-field kinematics measurements of planar or non-planar surfaces undergoing deformation (e.g. Hild \& Roux, 2006). It is a mathematical tool for assessing the spatial transformation (warping) between two digital images, so it is a way to measure deformation. Here, a 3D version of DIC is performed incrementally, between sequential loading stages. For each pair of images, the former (the reference image) is segmented into cubic REVs (correlation windows) and each of them is correlated with cubic regions of the same size within a predefined region (search window) in the latter (the deformed image). The correlation window with the highest normalised cross-correlation of the mean grey scale of each correlation window within the search window, represents the new state of the cube under investigation and, based on their spatial coordinates, displacement vectors are defined (e.g. Hall, 2006). Making a continuum hypothesis based on the finite-strain formulation (as explained in Roux \& Hild (2008)), the strain tensor is computed from the 3D displacement vectors. DIC belongs to the $3 \mathrm{D}$ continuum measurements and will give information about the incremental volumetric strain and the incremental maximum shear strain of the specimens (equations (1) and (2), respectively). The code TomoWarp2 was used in this work (Tudisco et al., 2015).

$$
\begin{aligned}
& \mathrm{d} \varepsilon_{\mathrm{vol}}=\mathrm{d} \varepsilon_{1}+\mathrm{d} \varepsilon_{2}+\mathrm{d} \varepsilon_{3} \\
& \mathrm{~d} \varepsilon_{\mathrm{s}_{\max }}=\frac{\sqrt{2}}{3} \sqrt{\left(\mathrm{d} \varepsilon_{1}-\mathrm{d} \varepsilon_{2}\right)^{2}+\left(\mathrm{d} \varepsilon_{1}-\varepsilon_{3}\right)^{2}+\left(\mathrm{d} \varepsilon_{2}-\mathrm{d} \varepsilon_{3}\right)^{2}}
\end{aligned}
$$

The strains of equations (1) and (2) have been calculated in terms of the principal values of the strain tensor and it should be mentioned that positive volumetric strains correspond to compaction. The mean values of the correlation of each increment are over $95 \%$, which defines the accuracy of the measurements and, since the standard deviation is small, there is a closeness of agreement among the whole set of measurements, proving that these measurements are sufficiently precise. It is clear that, while straining the specimen, it becomes less homogeneous and therefore the accuracy of the correlation reduces. However, when the shear band forms (after peak), the specimen does not undergo significant deformation outside the shear band and therefore the 
accuracy and precision of the measurements increase again. Additional confidence in the image analysis is given by the comparison of the measurement of total bulk volumetric strain (as it has been measured from the binary images, see Fig. 2(b)), with the average result from the DIC analysis, converted from an incremental to a total volumetric strain. The results are almost identical before localisation occurred and slightly deviated thereafter.

Figure 6 shows the evolution of incremental maximum shear and volumetric strains obtained from DIC analysis for test TCX1; the scales are different among the results, to highlight spatial variations at each increment. At the first increment, there is no significant increase in the maximum shear strain or the volumetric behaviour of the specimen; there is a region at the bottom of the specimen with higher shear strains accompanied by a slight compaction. The results suggest that the shear band initiates at that point, despite the low stresses that are applied at this loading increment. The shear strain increases at the bottom boundary (increment 2-3) and begins to propagate diagonally towards the top boundary (increment 3-4). The increase of shear strains is associated with a corresponding increase in compaction, as expected for a medium-dense specimen for high stresses. Until increment 3-4 there has been no record of any dilation throughout the whole specimen. As the maximum shear strain grows to a significant region of the specimen (increment 4-peak), the specimen starts to dilate inside a region of localised strain. The acceleration in dilation in this region occurs mainly post peak and finally accumulates within a band almost $1 \mathrm{~mm}\left(2\right.$ to $\left.3 D_{50}\right)$ thick. The region that is being sheared starts to narrow as soon as a significant number of grains were observed to break (increment peak-6); here the maximum shear strains reach values of slightly over $45 \%$.

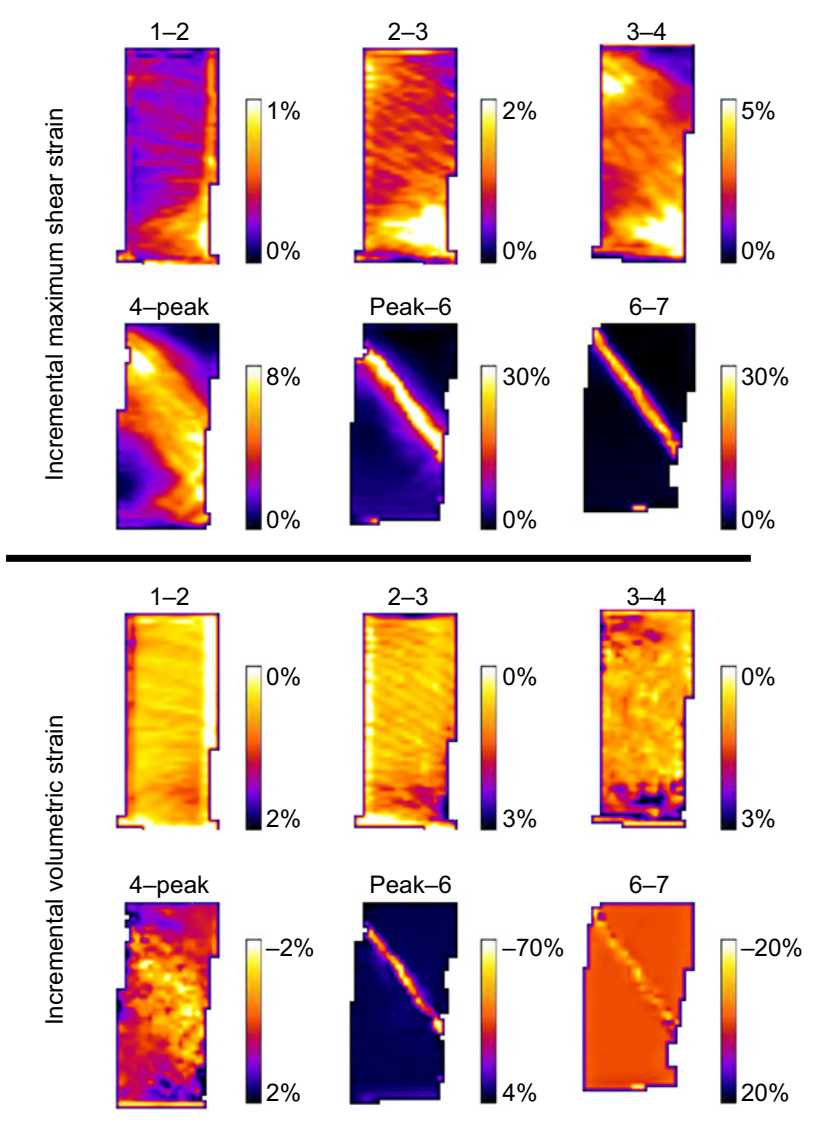

Fig. 6. DIC results of TCX1; top: incremental shear strain, bottom: incremental volumetric strain. (Note: the results are presented with different scale limits)
This shear band with very high shear strains is associated with very high localised dilation, while the rest of the specimen compacts by up to $4 \%$. Finally (increment 6-7), maximum shear strains of up to $25 \%$ localise into a narrower shear band $1.7 \mathrm{~mm}\left(4\right.$ to $\left.5 D_{50}\right)$ thick and, apart from within the shear band, the specimen does not undergo any significant volumetric change. It should be mentioned that the amount of breakage is such that the $D_{50}$ of the material before and after the test has remained the same.

From a visual comparison of the XCT images and the DIC results, it seems that in the centre of the shear band there is a zone of high comminution (see Fig. 3) related to the highest maximum shear strain and some dilation. Towards the outer region of the shear band, where the maximum shear strains reduce by $20 \%$ and there are no volumetric changes, there is a presence of both intact grains and fragments of bigger size. The inclination of the shear band appears to change slightly at the final two increments from $48^{\circ}$ to $53^{\circ}$ (relative to the horizontal plane). Similar results are found for TCX2, where the maximum shear strains also start to accumulate from the initial increment, forming a shear band of the same inclination and width.

Comparing the $7 \mathrm{MPa}$ high-confinement tests discussed so far, to the $100 \mathrm{kPa}$ low-confinement test (TCXL), which produces a peak behaviour with strong dilation, a shear band of similar inclination and thickness of 6 to $8 D_{50}$ is formed (Andò et al., 2013). There is no breakage present in TCXL, owing to the low confining stress. Alshibli \& Sture (1999) showed that the shear band thickness (relative to $D_{50}$ ) is not constant, as suggested by many researchers; instead, it decreases as $D_{50}$ increases. They measured a thickness of the shear band between 10 and $14 D_{50}$ for sands tested under plane strain conditions at confining stresses of $15 \mathrm{kPa}$ and $100 \mathrm{kPa}$. Tatsuoka et al. (1986: p. 74) showed that 'the average width of a shear band was smaller at a larger confining stress'. Taking into account that the sand in this work was tested at high confining stress $(7 \mathrm{MPa})$ and based on its $D_{50}$, it is reasonable that the thickness of the shear band varies between 4 and $5 D_{50}$.

\section{Grading analysis}

Being able to relate the localisation of strains to individual particle deformation, provides a further step towards linking the micro to the macro scale. A grading analysis provides information about the development of particle breakage during loading until failure. Several authors have used the shape of the grading curve to create continuum breakage mechanics (e.g. Hardin, 1985; Einav, 2007a, 2007b; Sun et al., 2015) models, to investigate compressibility and critical states. Even though these models consider the evolution of the grading curve, the experimental data on which they are built and/or calibrated are taken only before and after the test. In this study, the contribution of the XCT images and the image analysis give access to the study of the evolution of the grading at different loading stages during an experiment and at different regions of the specimen; to the best of the authors' knowledge, this has not been presented before.

Caicos particles are ellipsoids and, as Syvitski (1991) found for ellipsoids, the medium principal diameter $\left(D_{\text {med }}\right)$ mainly influences whether or not the particle will pass from a specific sieve opening. Therefore, a size descriptor had to be defined in this work to describe the particles' size and to relate the $3 \mathrm{D}$ measurements to conventional sieve analysis. Fonseca et al. (2014) also showed the importance of using one descriptor for particle size and how various measures correlate with the sieve curve and that it is common to use the true nominal diameter (diameter of sphere with same volume 
as the particle's) to describe the size of a particle. In this work each particle's diameter is initially defined based on the true nominal diameter, and this is related to the medium diameter of an ellipsoid of the same volume that has been prescribed to each particle (Fig. 7). After the lengths of the three principal diameters of the ellipsoid have been defined, these are compared to the true nominal diameter, taking into account the probability of the particular ellipsoid to pass from a sieve opening, depending on whether it is oblate or prolate (equation (3))

$$
\begin{aligned}
D_{\text {particle }} & =D_{\text {nominal }} \sqrt[3]{\frac{1}{\mathrm{FR}^{2} \mathrm{AR}}} \\
& =D_{\text {nominal }} \sqrt[3]{\frac{D_{\text {med }}^{2}}{D_{\text {min }} D_{\max }}}
\end{aligned}
$$

where the aspect ratio $\left(\mathrm{AR}=D_{\max } / D_{\min }\right)$ and the flatness ratio $\left(\mathrm{FR}=D_{\min } / D_{\text {med }}\right)$ are defined for the ellipsoid. It should be mentioned that, during the binarisation and segmentation of the images (Fig. 7), the amount of the fines that were not successfully binarised was calibrated with the mass of the specimen and fines were added to the grading curves.

This transformation (equation (3)) shifts the grading curve produced by image processing towards the sieve curve (Fig. 8(a)) and proves the importance of having a better size description for particle size. It will help compare results coming from image processing to more conventional experimental measurements and to previously published work (e.g. Nakata et al., 2001). It should be mentioned that the transformation was performed on all the specimens, producing consistently good results. Additionally, the XCT curves have an accuracy down to the voxel size (scanning parameters) and along with some expected noise from the images, the fines could be overestimated. The accuracy of the sieve curve would correspond to a $0 \cdot 0001 \mathrm{~g}$ mass measurement, underestimating the fines of lesser mass.

Figure 8 shows the evolution of grading in test TCX1, along with the relative breakage $\left(B_{\mathrm{r}}\right)$ and total breakage $\left(B_{\mathrm{t}}\right)$ of the specimen and the shear band exclusively. Einav (2007a) defines the breakage quantities as

$$
B_{\mathrm{r}}=\frac{B_{\mathrm{t}}}{B_{\mathrm{p}}}
$$
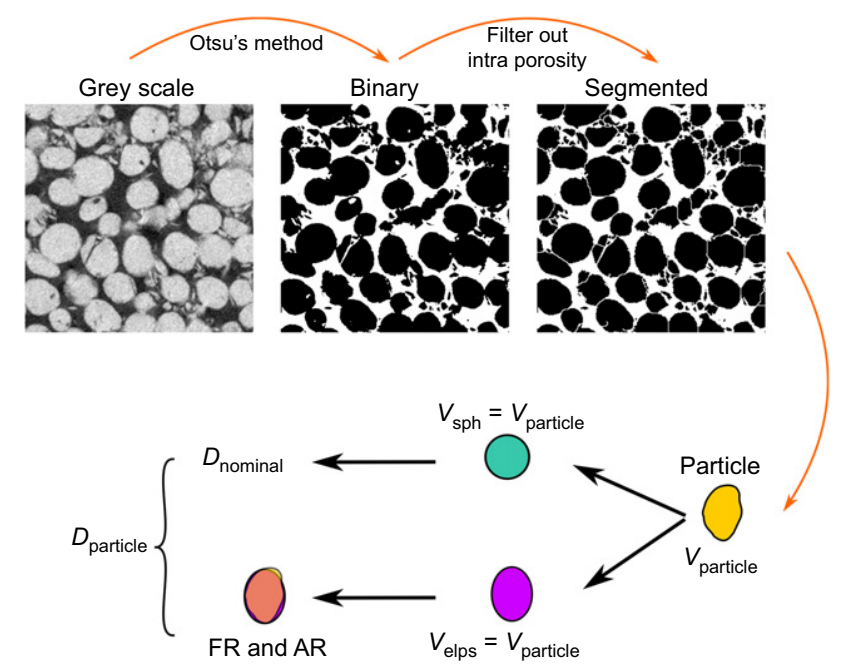

Fig. 7. Schematic diagram of the image analysis method used to obtain the grain size distribution

$$
\begin{aligned}
& B_{\mathrm{p}}=\int_{d_{\min }}^{d_{\max }}\left[F_{\mathrm{u}}(d)-F_{0}(d)\right] \mathrm{d}(\log d) \\
& B_{\mathrm{t}}=\int_{d_{\min }}^{d_{\max }}\left[F(d)-F_{0}(d)\right] \mathrm{d}(\log d)
\end{aligned}
$$

where $d_{\max }$ and $d_{\min }$ are the minimum and maximum particle diameters in the specimen after breakage has occurred, $F_{0}(d)$ is the initial grading curve, $F(d)$ the current and $F_{\mathrm{u}}(d)$ the assumed ultimate, with respect to the diameter $d$ of the particles (sieve opening). $B_{\mathrm{r}}$ denotes the relative breakage, $B_{\mathrm{p}}$ the potential breakage and $B_{\mathrm{t}}$ the total. Einav (2007a) proposed the following relationship for the ultimate grading curve

$$
F_{\mathrm{u}}(d)=\left(\frac{d}{d_{\max }}\right)^{0 \cdot 4}
$$

For $d$ close to $d_{\max }$, however, the curve of equation (7) lies below the experimentally measured grading curves. This is non-physical, as it indicates that breakage will lead to the creation of larger particles, therefore the following expression is used here, for the ultimate grading curve

$$
F_{\mathrm{u}}(d)=\min \left[\left(\frac{d}{d_{95}}\right)^{0.4}, 1\right]
$$

where $d_{95}$ is the diameter corresponding to $95 \%$ passing material.

Significant changes in the grading curves can be seen in the final three loading stages, indicating most breakage occurs post peak (Fig. 8(b)), even though from the DIC results more breakage was anticipated due to the intense increase of shear strains from at least two loading stages before peak (see Fig. 6). It is also interesting to observe that most breakage occurs between peak and immediately after (from $B_{\mathrm{t}}=1 \cdot 7 \%$ to $B_{\mathrm{t}}=3 \cdot 4 \%$ - Fig. $\left.8(\mathrm{~b})\right)$, whereas in the last two loading stages (residual strength), the change in the grading curve is less prominent (from $B_{\mathrm{t}}=3 \cdot 4 \%$ to $B_{\mathrm{t}}=4 \cdot 9 \%-\mathrm{Fig}$. $8(\mathrm{~b})$ ). It should be mentioned that additional breakage of particles into fragments smaller than $15 \mu \mathrm{m}$ (especially in the shear band) could have occurred, but these cannot be captured due to the limitations of the resolution of the images.

From the particle distribution function as shown in Fig. 8(c), it is clear that the biggest grains inside the specimen did not break, which can be attributed to the larger number of contacts of these grains. The grains that correspond to the peak of the histogram are those that mainly decrease in number, indicating breakage. It should be mentioned that these are the grains with a diameter close to $D_{50}$ and represent the majority of the grains. There is a clear increase in the number of fines produced; however, the number of fines (smaller than $150 \mu \mathrm{m}$ ) which broke into smaller fragments cannot be evaluated from this plot.

Figure 8(d) shows the gradings of the initial and final loading stages which are compared to the grading curves within the shear band (Fig. 8(e)) for the final loading stage and the theoretical ultimate $F_{\mathrm{u}}(d)$, in order to obtain $B_{\mathrm{r}}$. In the shear band, $B_{\mathrm{r}}$ is evaluated at $0 \cdot 3(16 \cdot 4 \%$ total breakage); this clearly indicates that the majority of breakage is concentrated in the shear band, when compared to the overall relative breakage that is 0.09 (4.9\% total breakage). Similar trends are observed in specimen TCX2, with a smaller overall percentage of total breakage $(2 \%)$, even though it was axially strained significantly more than TCX1. Both specimens exhibited the same theoretical breakage potential. There was no additional breakage between the last two loading stages at specimen TCX2, 


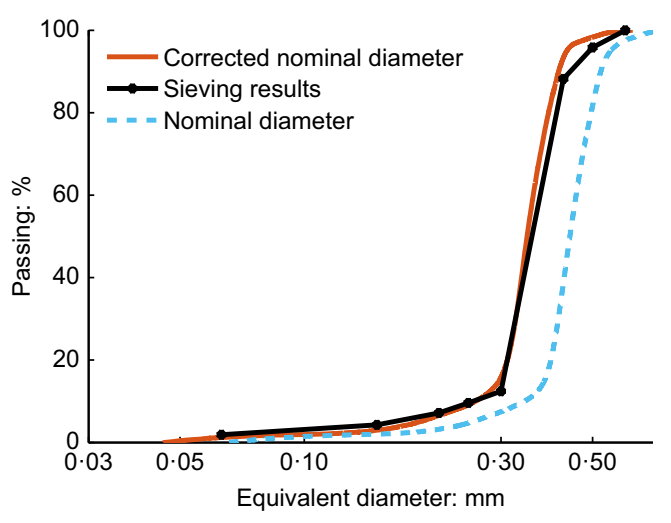

(a)

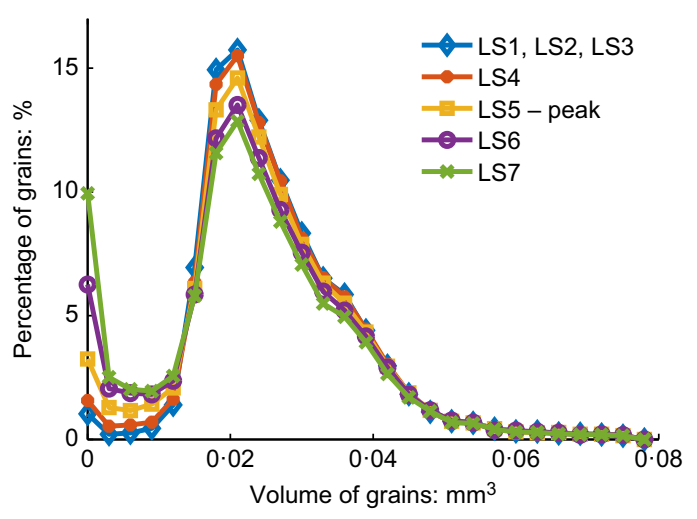

(c)

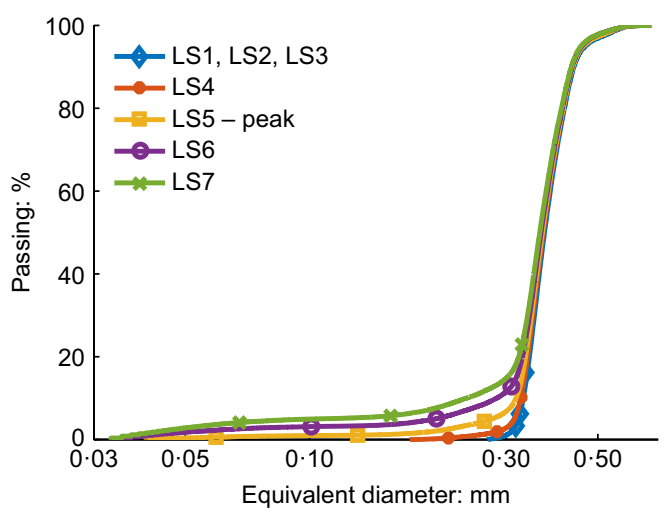

(b)

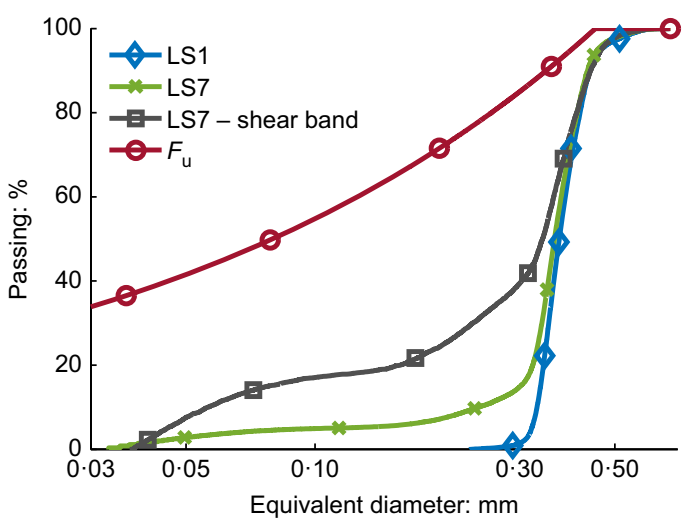

(d)

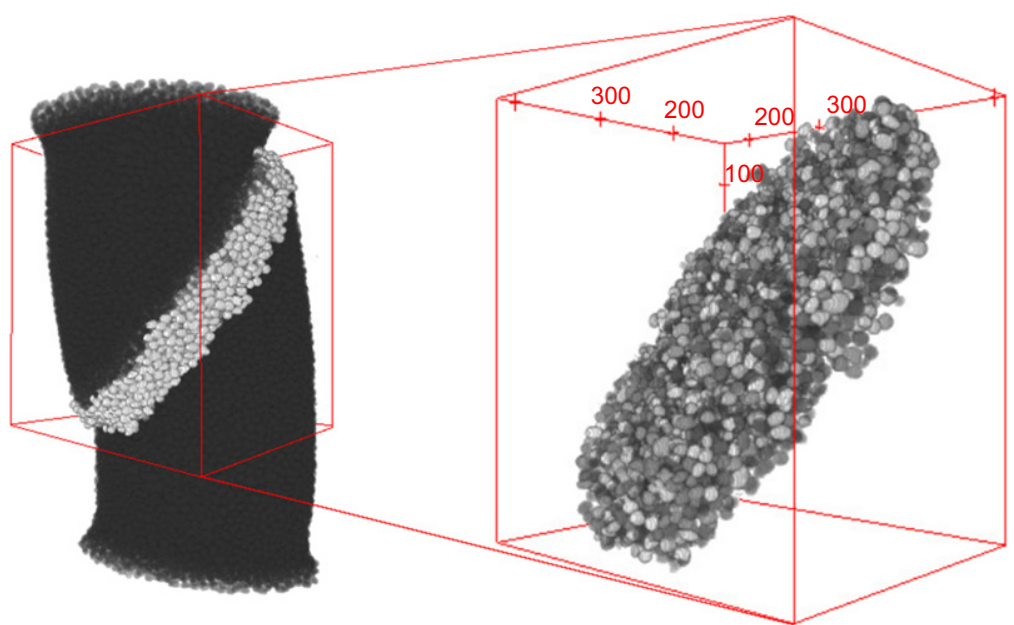

(e)

Fig. 8. Information on the evolution of breakage for test TCX1 from discrete image processing. (a) Determination of particle diameter transformation. (b) Grain size distribution evolution. (c) Histogram of evolution of volume of grains. (d) Determination of breakage. (e) 3D image of shear band at final loading stage (LS7). (Note that the grading curve from the XCT analysis is calculated based on the number of particles passing each virtual sieve opening and converted into a mass equivalent curve, as described in the text)

which indicates that the fully formed shear band would allow the two halves of the specimen to translate relative to each other instead of causing more breakage and would cause the specimen within the shear band to compact (Fig. 5). These observations are in agreement with the DIC results, and the lower amount of breakage of TXC2 in comparison to TXC1 can be explained by the difference in post-peak volumetric behaviour (compaction as opposed to dilation, Fig. 5).

Figure 9 shows the distribution of $B_{\mathrm{r}}$ and $B_{\mathrm{t}}$ in test TCX1. It should be mentioned that the elementary volume used in this algorithm was twice as big as the REV used for the porosity maps and the DIC, in order to include more grains and to result in a more detailed grain size distribution for each elementary volume. Even though it is not an REV, for brevity it will be referred to as an REV in this section. After careful inspection, it was found that the grain size distribution for each REV in the initial loading stage has the same shape as the distribution curve of the whole specimen. This confirms the homogeneity of the specimen and so, for the creation of the breakage maps of Fig. 9, the grading curve of the whole initial specimen is used as the $F_{0}$. Consequently, $B_{\mathrm{p}}$ is constant throughout the whole specimen (equation (5)) and so $B_{\mathrm{t}}$ is always proportional to $B_{\mathrm{r}}$ (equation (4)), allowing the same colour maps to be used for both relative and total 

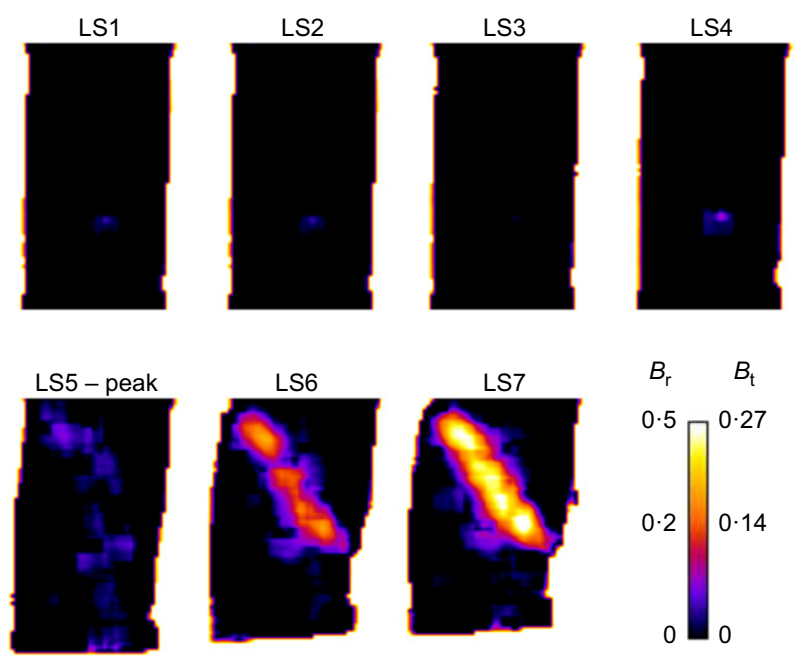

Fig. 9. Distribution of total breakage $\left(B_{t}\right)$ and relative breakage $\left(B_{r}\right)$ for test TCX1

breakage. These images enable the comparison of the continuum image processing with the discrete and provide information about the spatial distribution of breakage and deformation. A link thus can be made between strains and breakage, based on experimental measurements, which has not been presented before and could facilitate a direct comparison between experimental data and constitutive modelling.

It is clear from Fig. 9 that breakage occurs throughout the whole specimen starting from peak (LS5) onwards, with a higher percentage within and around the shear band. Before peak stress (LS5), there is very little non-localised breakage, accompanied by a slight increase in shear strain and only compaction. At peak, there is an important increase of the relative breakage; however, it does not seem to localise yet, even though the DIC analysis shows clear localised dilative and maximum shear strains. From the comparison of the porosity distribution between the initial loading stage and peak, there is an overall reduction of porosity. Significant breakage is measured post peak, along the shear band and there seems to be an area affected by breakage at the bottom of the specimen, which is where the loading was applied. It should be mentioned that at the boundaries it is expected that some breakage might have occurred (especially at the ends of the shear band), which could not be measured using this algorithm owing to the small number of grains present in the REVs.

\section{CONCLUSIONS}

In this paper, XCT was used to visualise entire specimens of sand, under high-confinement triaxial compression tests. The acquired images were processed and measurements were made on breakage, local variations of porosity, volumetric strain, maximum shear strain, grading and relative breakage. The material used for this study was Caicos ooids - a sand with very rounded particles. The specimens have a mean diameter of $380 \mu \mathrm{m}$, a uniform grading and a medium initial relative density. The macroscopic response of the specimens shows an overall compactive behaviour under high confinement, consistent among various tests.

Regarding the image processing, initially, porosity maps were created based on the density distribution of the grey scale images. The specimens prior to shearing are rather homogeneous and, as the deviatoric loading increases, there is an overall reduction of the porosity, with a concentration at the bottom half of the specimen. At the final two loading stages (post peak), a band with increased porosity appears in test TCX1, indicating some dilation, and this area coincides with where breakage mainly concentrates; whereas test TCX2 is mainly exhibiting a constant decrease of porosity. Interestingly, the overall DIC analysis shows that the shear band initiates at the initial increment from a single point at the bottom edge of the specimen and propagates to the top opposite edge, until finally it develops into a full shear band post peak. Before peak there is no evidence of any dilation, confirming that the small amount of breakage is a result of the high compaction of the specimens. The majority of breakage occurs post peak and is a result of large shear strains under high confining stresses. Post peak, there is significant shearing, localised along a single band of 4 to $5 D_{50}$, in the centre of which dilation concentrates within a narrower band of 2 to $3 D_{50}$. Right after peak, the rest of the specimen is slightly compacting and develops low maximum shear strains, whereas at the final increment all of the strains develop only within the shear band. The overall breakage of the specimens is close to $5 \%$, which is rather low and so the $D_{50}$ remains constant, but the images enable the investigation of breakage of specific regions, where in some cases it reached more than $30 \%$. Most of the breakage concentrates along the shear band and at the bottom half of the specimen, indicating a boundary effect, due to the application of loading. Particles start to break as soon as the deviatoric load is applied, which indicates that the high stresses caused the material to compact and to strongly shear. As soon as the particles start to break and both breakage and strains localise (peak and post peak) the specimen starts to dilate in the shear band, allowing the development of higher maximum shear strains and more breakage. Interestingly, the two mechanisms of particle rearrangement and particle breakage coexist within the shear band. Test TCX1 exhibits more breakage than test TCX2, even though it is axially strained less. In addition, TCX1 shows an increase of the porosity in the shear band, where most of the breakage occurs, potentially explained by the creation of more particles, which are also more angular than the intact particles. When there is no additional breakage and the specimen has already undergone sufficient dilation, the specimen starts to compact again within the shear band. Overall, two independent mechanisms were observed in coexistence, leading to areas within the specimen where both volumetric dilation and compaction were present.

It is the intention of the authors to extend this research by investigating the breakage of granular materials occurring along other stress paths, such as one-dimensional (oedometric) compression, using the same tools and similar techniques.

\section{ACKNOWLEDGEMENTS}

The authors would like to thank Pascal Charrier for his valuable help during the XCT. Laboratoire $3 \mathrm{SR}$ is part of the LabEx Tec 21 (Investissements dAvenir - grant agreement nANR-11-LABX-0030). Z. Karatza would like to thank the UK Engineering and Physical Sciences Research Council (EPSRC) DTP PhD studentship and the International Fine Particles Research Institute (IFPRI) for funding her PhD research. S.-A. Papanicolopulos acknowledges the support received from the People Programme (Marie Curie Actions) of the European Union's Seventh Framework Programme (FP7/2007-2013) under REA grant agreement no. 618096. G. Viggiani acknowledges the support received from the Royal Academy of Engineering through a Distinguished Visiting Fellowship. 
NOTATION

$\begin{aligned} B_{\mathrm{p}} & \text { potential breakage } \\ B_{\mathrm{r}} & \text { relative breakage } \\ B_{\mathrm{t}} & \text { total breakage } \\ C_{\mathrm{U}}=D_{60} / D_{10} & \text { coefficient of uniformity } \\ D_{\mathrm{max}} & \text { maximum particle Feret diameter } \\ D_{\mathrm{med}} & \text { medium principal particle diameter } \\ D_{\min } & \text { minimum particle Feret diameter } \\ D_{\text {nominal }} & \text { nominal particle diameter } \\ D_{\text {particle }} \text { or } d & \text { particle diameter } \\ D_{10} & \text { diameter of } 10 \% \text { of particles passing } \\ D_{50} & \text { mean particle diameter } \\ D_{60} & \text { diameter of } 60 \% \text { of particles passing } \\ d_{\max } & \text { maximum particle diameter in specimen } \\ d_{\mathrm{min}} & \text { minimum particle diameter in specimen } \\ \mathrm{d} \varepsilon_{\mathrm{smax}} & \text { incremental maximum shear strain } \\ \mathrm{d} \varepsilon_{\mathrm{vol}} & \text { incremental volumetric strain } \\ \mathrm{d} \varepsilon_{1} & \text { incremental major principal strain } \\ \mathrm{d} \varepsilon_{2} & \text { incremental intermediate principal strain } \\ \mathrm{d} \varepsilon_{3} & \text { incremental minor principal strain } \\ d_{95} & \text { diameter of } 95 \% \text { of particles passing } \\ e_{\max } & \text { maximum void ratio } \\ e_{\mathrm{min}} & \text { minimum void ratio } \\ F & \text { current grading curve } \\ F_{\mathrm{u}} & \text { ultimate grading curve } \\ F_{0} & \text { initial grading curve } \\ p & \text { mean stress } \\ q & \text { stress deviator } \\ V_{\mathrm{elps}} & \text { volume of ellipse } \\ V_{\mathrm{particle}} & \text { volume of particle } \\ V_{\mathrm{sph}} & \text { volume of sphere } \\ \varepsilon_{\mathrm{a}} & \text { axial strain } \\ \varepsilon_{\mathrm{vol}} & \text { volumetric strain } \\ \sigma_{1} & \text { major principal stress } \\ \sigma_{3} & \text { minor principal stress } \\ & \end{aligned}$

\section{REFERENCES}

Alikarami, R., Andò, E., Gkiousas-Kapnisis, M., Torabi, A. \& Viggiani, G. (2015). Strain localisation and grain breakage in sand under shearing at high mean stress: insights from in situ x-ray tomography. Acta Geotechnica 10, No. 1, 15-30.

Alshibli, K. A. \& Sture, S. (1999). Sand shear band thickness measurements by digital imaging techniques. J. Comput. Civ. Engng 13, No. 2, 109-109.

Andò, E. (2013). Experimental investigation of micro-structural changes in deforming granular media using X-ray tomography. $\mathrm{PhD}$ thesis, Université de Grenoble, Grenoble, France.

Andò, E., Hall, S. A., Viggiani, G., Desrues, J. \& Bésuelle, P. (2012). Grain-scale experimental investigation of localised deformation in sand: a discrete particle tracking approach. Acta Geotechnica 7, No. 1, 1-13.

Andò, E., Viggiani, G., Hall, S. A. \& Desrues, J. (2013). Experimental micro-mechanics of granular media studied by $\mathrm{X}$-ray tomography: recent results and challenges. Géotechnique Lett. 3, No. 3, 142-146, http://dx.doi.org/10.1680/geolett.13. 00036.

Arslan, H., Baykal, G. \& Sture, S. (2009). Analysis of the influence of crushing on the behaviour of granular materials under shear. Granular Matter 11, No. 2, 87-96.

ASTM (2014) D4253-14: Standard test methods for maximum index density and unit weight of soils using a vibratory table. West Conshohocken, PA, USA: ASTM International.

Bishop, A. W. \& Henkel, D. J. (1962). The measurement of soil properties in the triaxial test, 2nd edn. London, UK: English Language Book Society and Edward Arnold.

Bolton, M. D. (1986). The strength and dilatancy of sands. Géotechnique 36, No. 1, 65-78, http://dx.doi.org/10.1680/geot. 1986.36.1.65.

Cil, M. B. \& Alshibli, K. A. (2014). 3D evolution of sand fracture under 1D compression. Géotechnique 64, No. 5, 351-364, http://dx.doi.org/10.1680/geot.13.P.119.

Colliat-Dangus, J. L., Desrue, J. \& Foray, P. (1988). Triaxial testing of granular soil under elevated cell pressure. In Advanced triaxial testing for soil and rock (eds R. T. Donaghe, R. C. Chaney and
M. L. Silver), STP 977, pp. 290-310. West Conshohocken, PA, USA: ASTM International.

Coop, M. R., Sorensen, K. K., Bodas Freitas, T. \& Georgoutsos, G. (2004). Particle breakage during shearing of a carbonate sand. Géotechnique 54, No. 3, 157-163, http://dx.doi.org/ 10.1680/geot.2004.54.3.157.

Desrues, J., Chambon, R., Mokni, M. \& Mazerolle, F. (1996). Void ratio evolution inside shear bands in triaxial sand specimens studied by computed tomography. Géotechnique 46, No. 3, 529-546, http://dx.doi.org/10.1680/geot.1996.46.3.529.

Einav, I. (2007a). Breakage mechanics part I: theory. J. Mech. Phys. Solids 55, No. 6, 1274-1297.

Einav, I. (2007b). Breakage mechanics part II: modelling granular materials. J. Mech. Phys. Solids 55, No. 6, 1298-1320.

Fonseca, J., O'Sullivan, C., Coop, M. R. \& Lee, P. D. (2013a). Quantifying the evolution of soil fabric during shearing using directional parameters. Géotechnique 63, No. 6, 487-499, http://dx.doi.org/10.1680/geot.12.P.003.

Fonseca, J., O'Sullivan, C., Coop, M. R. \& Lee, P. D. (2013b). Quantifying the evolution of soil fabric during shearing using scalar parameters. Géotechnique 63, No. 10, 818-829, http://dx. doi.org/10.1680/geot.11.P.150.

Fonseca, J., Sim, W., Shire, T. \& O'Sullivan, C. (2014). Microstructural analysis of sands with varying degrees of internal stability. Géotechnique 64, No. 5, 405-411, http://dx. doi.org/10.1680/geot.13.T.014.

Gkiousas-Kapnisis, M., Andò, E., Tengattini, A., Einav, I. \& Viggiani, G. (2015). Development of image analysis tools to evaluate in-situ evolution of the grain size distribution in sand subjected to breakage. In Bifurcation and degradation in the new millennium: Proceedings of the 10th international workshop on bifurcation and degradation in geomaterials (eds K.T. Chau and J. Zhao), pp. 253-258. Cham, Switzerland: Springer.

Hall, S. A. (2006). A methodology for 7D warping and deformation monitoring using time-lapse seismic data. Geophysics 71, No. 4, $21-31$.

Hardin, B. O. (1985). Crushing of soil particles. J. Geotech. Engng 111, No. 10, 1177-1192.

Hild, F. \& Roux, S. (2006). Digital image correlation: from displacement measurement to identification of elastic properties - a review. Strain 42, No. 2, 69-80.

Kikumoto, M., Muir Wood, D. \& Russell, A. (2010). Particle crushing and deformation behaviour. Soils Found. 50, No. 4, $547-563$.

Lloyd, R. M., Perkins, R. D. \& Kerr, S. D. (1987). Beach and shoreface ooid deposition on shallow interior banks, Turks and Caicos Islands, British West Indies. J. Sedimentary Petrol. 57, No. 6, 976-982.

McDowell, G. R., Bolton, M. D. \& Robertson, D. (1996). The fractal crushing of granular materials. Int. J. Mech. Phys. Solids 44, No. 12, 2079-2102.

Nakata, Y., Hyodo, M., Hyde, A. F. L., Kato, Y. \& Murata, H. (2001). Microscopic particle crushing of sand subjected to high pressure one-dimensional compression. Soils Found. 41, No. 1, 69-82.

Oda, M., Takemura, T. \& Takahashi, M. (2004). Microstructure in shear band observed by microfocus X-ray computed tomography. Géotechnique 54, No. 8, 539-542, http://dx.doi. org/10.1680/geot.2004.54.8.539.

Razavi, M., Muhunthan, B. \& Al Hattamleh, O. (2006). Representative elementary volume analysis of sands using X-ray computed tomography. Geotech. Testing J. 30, No. 3, $1-8$.

Roscoe, K., Schofield, A. N. \& Wroth, C. (1958). On the yielding of soils. Soils Found. 54, No. 3, 451-461.

Roux, S. \& Hild, F. (2008). Digital image mechanical identification (DIMI). Expl Mech., Soc. Expl Mech. 48, No. 4, 495-508.

Schluter, S., Sheppard, A., Brown, K. \& Wildenschild, D. (2014). Image processing of multiphase images obtained via X-ray microtomography: A review. Water Resources Res. 50, No. 4, 3615-3639.

Sun, Y. F., Xiao, Y. \& Hanif, K. F. (2015). Compressibility dependence on grain size distribution and relative density in sands. Technol. Sci. 58, No. 3, 443-448. 
Syvitski, J. P. M. (1991). Principles, methods and application of particle size analysis, 1st edn. Cambridge, UK: Cambridge University Press.

Tatsuoka, F., Sakamoto, M., Kawamura, T. \& Fukushima, S. (1986). Strength and deformation characteristics of sand in plane strain compression at extremely low pressures. Soils Found. 26, No. 1, 65-84.

Tengattini, A., Das, A. \& Einav, I. (2015). A theory predicting breakage dependence of critical state in sand. In Geomechanics from micro to macro (eds K. Soga, K. Kumar, G. Biscontin and M. Kuo), pp. 695-698. London, UK: Taylor \& Francis.

Tengattini, A., Das, A. \& Einav, I. (2016). A constitutive modelling framework predicting critical state in sand undergoing crushing and dilation. Géotechnique 66, No. 9, 695-710, http://dx.doi. org/10.1680/jgeot.14.P.164.
Tudisco, E., Hall, S. A., Charalampidou, E. M., Kardjilov, N., Hilger, A. \& Sone, H. (2015). Full-field measurements of strain localisation in sandstone by neutron tomography and 3D-volumetric digital image correlation. Physics Procedia 69, 509-515, https://doi.org/10.1016/j.phpro.2015.07. 072.

Wildenschild, D. \& Sheppard, A. P. (2013). X-ray imaging and analysis techniques for quantifying pore-scale structure and processes in subsurface porous medium systems. Adv. Water Resources 51, 217-246, https://doi.org/10.1016/j.advwatres. 2012.07.018.

Zhao, B., Wang, J., Coop, M. R., Viggiani, G. \& Jiang, M. (2015). An investigation of single sand particle fracture using X-ray micro-tomography. Géotechnique 65, No. 8, 625-641, http://dx. doi.org/10.1680/geot.4.P.157. 